\title{
ACCURACY OF THE MULTIPLE STATIONS PASSIVE OPTOELECTRONIC RANGE-FINDING SYSTEM
}

\begin{abstract}
V. Čech*
Abstract: Localization of non-cooperative moving objects (targets) is a very significant technical problem, which has several military and security applications. In praxis, passive and active localization systems are being used. Our team has been carrying out research and development of passive optoelectronic rangefinding systems (POERFS) for 15 years. In this paper, we provide basic information on the precision/accuracy estimation method of multiple stations POERFS.
\end{abstract}

Keywords: Passive optoelectronic range-finding system (POERFS), multiple stations POERFS, rangefinder (RF), target position localization system, accuracy.

\section{Localization task and Passive optoelectronic range-finding systems}

The topographical coordinates of an object of interest (the target), which is represented by one contractual point $\mathrm{T}$, need to be determined indirectly in many cases that occur in practice because an access to respectively the target and the target point $\mathrm{T}$ is disabled due to miscellaneous reasons at a given time. Hereafter we will confine to methods that make use of specialized technical equipment - localization systems (range -finding systems or rangefinders) to determine coordinates of the target point $\mathrm{T}$ (Cech 2011). Localization systems are a special kind of sensor systems. Localization systems (rangefinders) fall into two categories, i.e. active and passive localization systems (rangefinders).

We have been carrying out research and development of passive optoelectronic range-finding systems (rangefinders) - POERFS on a long-term basis. You can find the basic information in Cech 2011. We distinguish single station and two or multiple stations PORFS. We have published basic information about single station POERFS in Cech 2013 and about multiple stations POERFS in Cech 2018, Fig. 1, 2.

The point $\mathrm{P}_{\mathrm{RF}}=\mathrm{CU}_{\mathrm{i}}, \mathrm{i}=0,1, \ldots$ Fig. 2 represents a contractual position of the rangefinder in the topographical coordinate system, $\mathrm{D}_{\mathrm{T}}$ is the target slant range measured by means of the rangefinder. This value $D_{T}$ represents the estimate of the real slant range of the target $D_{T 0}$ that is equal contractually to the distance of points $\mathrm{P}_{\mathrm{RF}}$ and T. The angle $\varepsilon_{\mathrm{T}}$ is the measured estimate of the elevation of the target $\varepsilon_{\mathrm{T} 0}$ and the angle $\alpha_{\mathrm{T}}$ is the measured estimate of the target azimuth $\alpha_{\mathrm{T} 0}$. The coordinates $(\mathrm{D}, \varepsilon, \alpha)$ are relative spherical coordinates towards the contractual position of the rangefinder which is represented by the point $\mathrm{P}_{\mathrm{RF}}$.

Multiple Stations POERFS (Fig. 3) contains at least two camera units (CU), central computer, operator's workplace and operator. The system sends measured and extrapolated information about the position of the target (Fig. 1) into central dispatching, which determines their further use. At the same time, it controls the activity of the system operator.

At a given station at least one camera unit CU is placed. Stations with camera units can be placed e.g. up to the perimeter of the protected object (Cech 2018). The camera unit is a special type of theodolite - Fig. 1 , whose task it is to measure angular coordinators of a target. In most applications the accuracy of 
measurement cc. $0.1 \mathrm{mrad}$ (cc $20 \mathrm{arc} \mathrm{sec}$ ) is sufficient (Cech 2018). The image of the object is recorded by

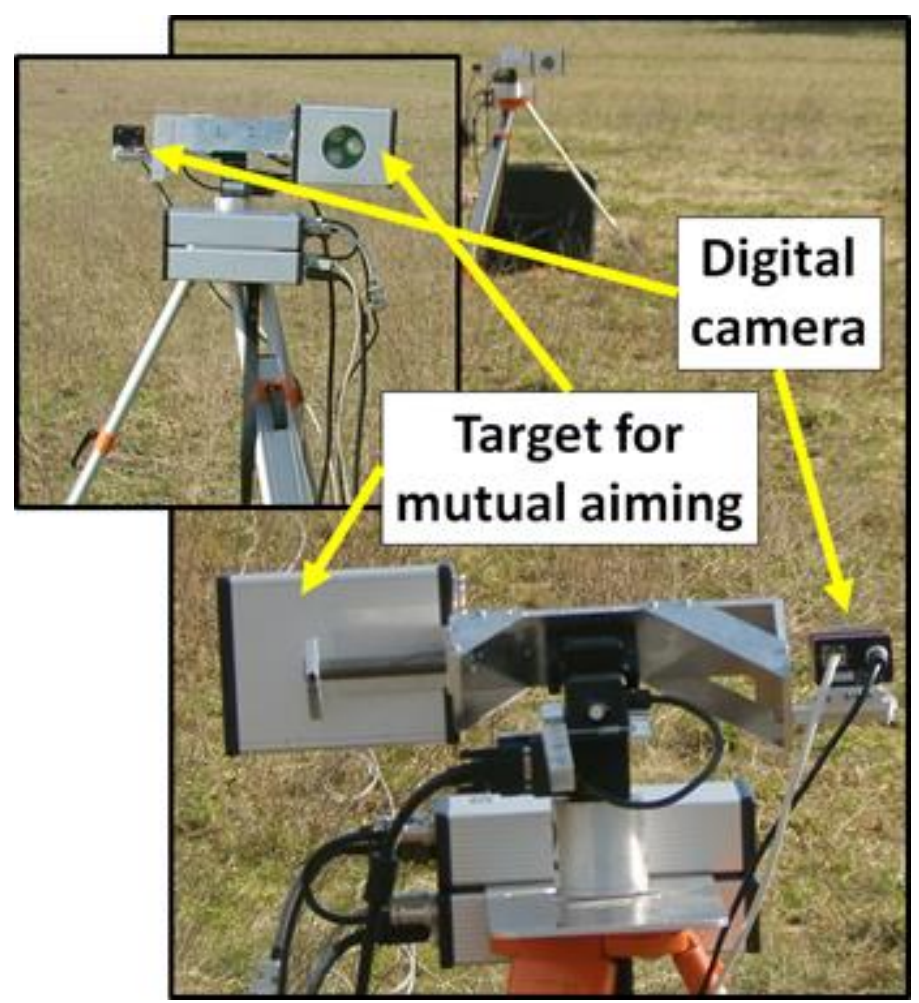

Fig. 1: Two measurement camera units CU1 and CU2 in position for the mutual aiming

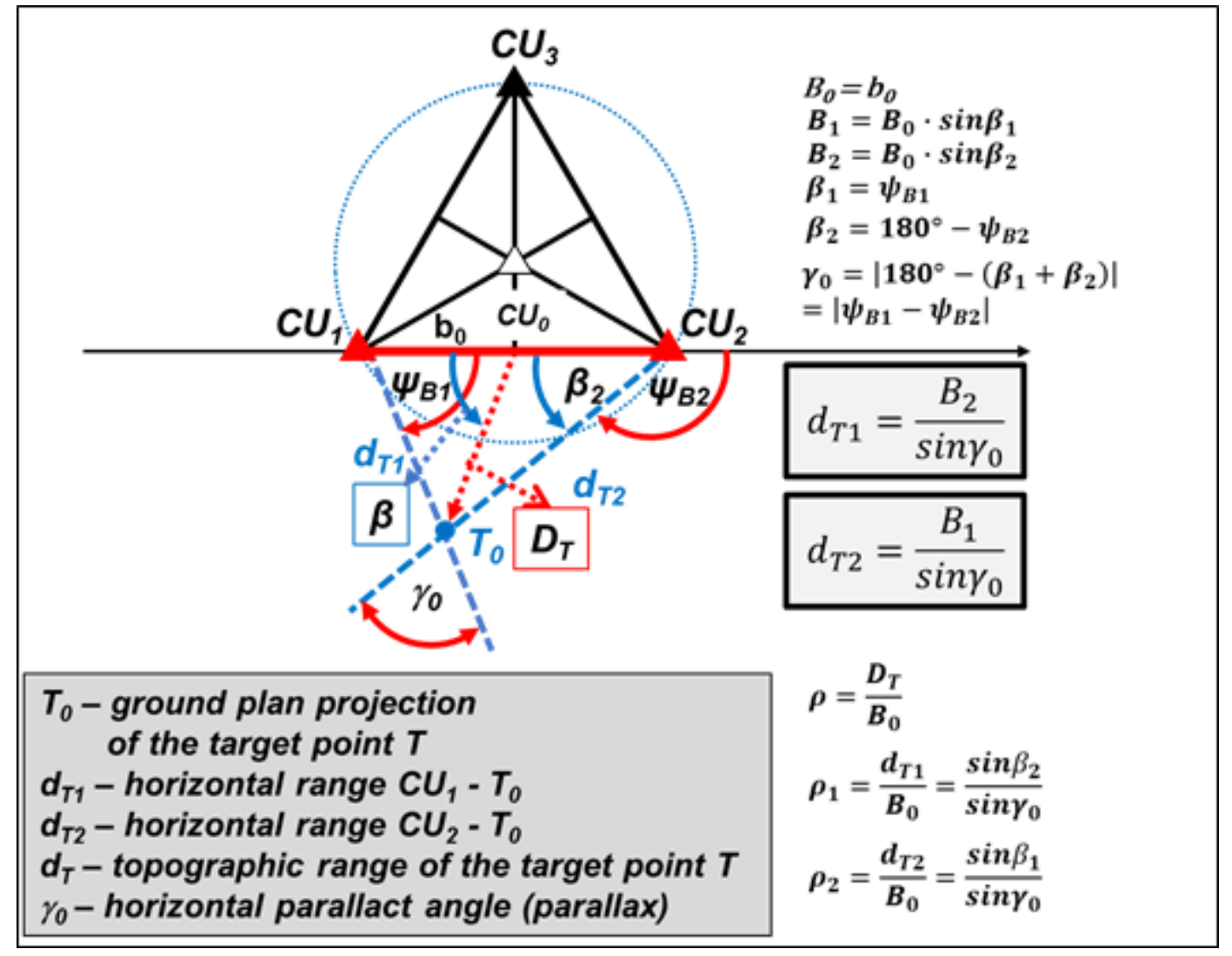

Fig. 2: Principle of the estimation of target ranges $d_{T 1}$ and $d_{T 2}$. (Cech 2018) 


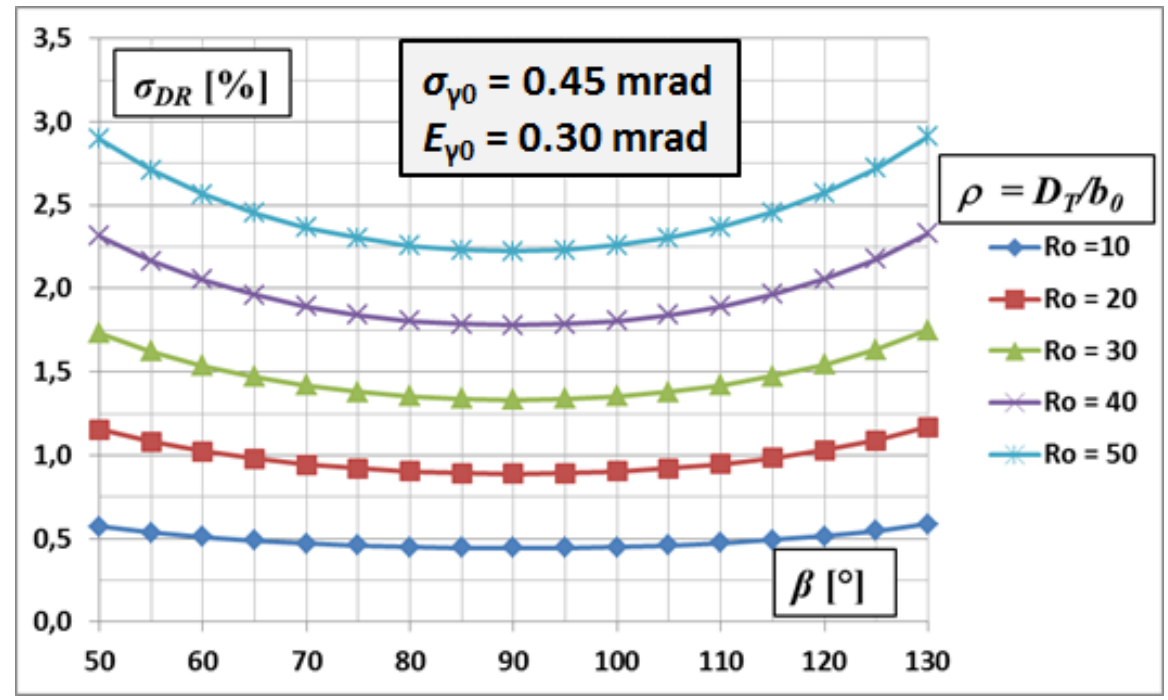

Fig. 3: Example of accuracy of two stations POERFS (Cech 2018)

at least one digital camera or thermal imaging camera of CU. Cameras are placed on a special Pan and Tilt Device (PTD) or a console.

Omnidirectional measurement of target position is very often required (Cech 2018). To fulfil this requirement, it is necessary to suitably place at least three measurement camera units - MCU (CU1, CU2 and CU3 on Fig. 2) and at least one surveillance/spotting camera unit - SCU (CU0 on Fig. 2).

We simplify the task and assume that the CU1, CU2 and T points lie in the common horizontal plane Fig. 2. The calculation principle of the horizontal range of the target $\mathrm{D}_{\mathrm{T}}$ and angle $\beta$ is clear from Fig. 2 (Cech 2018). From the position measurements of target point $T$ from two camera units CU1 and CU2 horizontal angles $\psi_{\mathrm{B} 1}$ and $\psi_{\mathrm{B} 2}$ are calculated. From the measured size of the horizontal base $\mathrm{b}_{0}=\mathrm{B}_{0}$ and the angles $\psi_{\mathrm{B} 1}$ and $\psi_{\mathrm{B} 2}$ horizontal ranges $\mathrm{d}_{\mathrm{T} 1}$ and $\mathrm{d}_{\mathrm{T} 2}$ are calculated. Then we can determine $\mathrm{D}_{\mathrm{T}}$ and $\beta$ (Kraus 2000).

\section{Accuracy of two stations POERFS}

We use the usual procedure to derive the relationship for standard deviations (Cech 2011). For greater distances $\mathrm{D}_{\mathrm{T}}$ is true $\mathrm{D}_{\mathrm{T}}=\mathrm{d}_{\mathrm{T}} \approx \mathrm{d}_{\mathrm{T} 1} \approx \mathrm{d}_{\mathrm{T} 2}$, Fig. 2. The relationship for $\mathrm{d}_{\mathrm{T}}=\mathrm{d}_{\mathrm{T} 1}$ is used as the default to derive the following formula for relative value of standard deviation of horizontal range $d_{T}$

$$
\sigma_{D R}=\frac{\sigma_{D}}{d_{T}} \approx \sqrt{\left[\sigma_{D R, 1}^{2}+\sigma_{D R, 2}^{2}+\sigma_{D R, 3}^{2}\right]},
$$

where

$$
\sigma_{D R, 1}=\frac{\sigma_{b}}{b_{0}}
$$

is the relative value of standard deviation $\sigma_{b}$ which characterize measurement precision of base $b_{0}$ Fig. 2 . There is no big problem to keep $\sigma_{\mathrm{DR}, 1}$ under 0,01 . In this case, the contribution of $\sigma_{\mathrm{DR}, 1}$ to the whole $\sigma_{\mathrm{DR}}$ is small.

$$
\sigma_{D R, 2} \approx\left|\cot \left(\beta_{2}\right)\right| \cdot \sigma_{\alpha},
$$

represents the influence of measurement precision of angles $\beta_{2}$ and $\psi_{\mathrm{B} 2}$, respectively. The size of the angle $\beta_{2}$ depends on the angle $\beta$ and it lies usually between $50^{\circ}$ and $130^{\circ}$ for the greater ranges $D_{T}$ of the target and thus the contribution $\sigma_{\mathrm{DR}, 2}$ to the whole $\sigma_{\mathrm{DR}}$ is small (Fig. 3).

$$
\sigma_{D R, 3} \approx\left|\cot \left(\gamma_{0}\right)\right| \cdot \sigma_{\gamma 0},
$$

and

$$
\sigma_{\gamma 0}=\sqrt{K_{\gamma}} \cdot \sigma_{\alpha},
$$


$\mathrm{K}_{\gamma}=1.0$ to 2.0 is constant. The magnitude of this constant depends on how the angles $\psi_{\mathrm{B} 1}$ and $\psi_{\mathrm{B} 2}$ are measured.

If these angles are measured completely independent of each other, then $\mathbf{K}_{\gamma}=2.0$.

If these angles are measured entirely in master - slave mode, then $\mathrm{K}_{\gamma}$ approaches the value 1.0. In this case, the mutual aiming method is always used to set zero values of angles $\psi_{\mathrm{B} 1}$ and $\psi_{\mathrm{B} 2}$ Fig. 1.

$\sigma_{\alpha}$ is standard deviation that characterizes the precision of the measurement of angles $\psi_{\mathrm{B} 1}$ and $\psi_{\mathrm{B} 2}$.

For larger distances $\mathrm{D}_{\mathrm{T}}$, the parallax $\gamma$ or $\gamma_{0}$ is very small and therefore $\sigma_{\mathrm{DR}, 3}$ has a decisive influence on the whole $\sigma_{\mathrm{DR}}$, then $\sigma_{\mathrm{DR}} \approx \sigma_{\mathrm{DR}, 3}$. Obviously, the $\mathrm{K}_{\gamma}$ and $\sigma_{\alpha}$ variables have a decisive influence on the precision of the range measurements.

POERFS enable to measure in master - slave mode and therefore can be achieved $\mathrm{K}_{\gamma} \rightarrow 1.0$.

The size of the standard deviation $\sigma_{\alpha}$ depends both on the measured accuracy of the pan and tilt device angles and on the accuracy of the position evaluation of the target point $\mathrm{T}$ in the images of both digital cameras. The size of standard deviation $\sigma_{\alpha}$ also depends on atmospheric conditions, scene illumination, and target image contrast to the image of its surroundings (Cech 2011, Roggeman 1996).

To illustrate the accuracy of the system we provide an example - Fig. 3. The typical size of a standard deviation of parallax $\sigma_{\gamma 0}$ is cc. $0.45 \mathrm{mrad}$ (probable error $\mathrm{E}_{\gamma 0}=0.30 \mathrm{mrad}$ ), which depends on the accuracy of determining the angles $\psi_{\mathrm{B} 1}$ and $\psi_{\mathrm{B} 2}$. Relative value of standard deviation of horizontal target range $\sigma_{\mathrm{DR}}$ then depends on the selected size of the horizontal base $b_{0}=B_{0}$. To achieve sufficient measurement accuracy of the target range $\mathrm{D}_{\mathrm{T}}$ it is necessary to choose $\mathrm{b}_{0}=\mathrm{D}_{\text {Tmax }} /(10$ to 50$)$, where $\mathrm{D}_{\text {Tmax }}$ is a selected maximum target range.

The above analysis applies similarly to type A and type B uncertainties as well as for overall measurement uncertainties (ISO Guide of Uncertainties in Measurement (GUM)). Type A uncertainties are also referred to as consistency or precision (ISO $5725-1$ ) or random errors of measurement. Type B uncertainties are also referred to as accuracy (ISO 5725 - 1) or statistical bias or pseudo - random errors or systematic errors of measurement. Overall uncertainties are also referred to as overall/total precision of measurement.

\section{Conclusion}

Multiple stations POERFS (Cech 2018) have basically identical utility features as single station POERFS, which we analyzed in Cech 2013. The comparison of the qualities of POERFS with the qualities of laser rangefinders - LRF is presented in Cech 2013 and 2018.

Effectiveness of POERFS and LRFs is decreased by attenuation of radiation and turbulence of atmosphere (Roggeman 1996).

Further information about multiple stations POERFS will be published in the following period.

\section{Acknowledgement}

This work was supported by Ministry of education, science, research and sport of the Slovak republic as a part of the industrial research project "Passive optoelectronic localization system (POERF)" (decision No. 2018/14626:9-26CO).

\section{References}

Cech, V. and J. Jevicky. (2011) Research and development of the passive optoelectronic rangefinder, in Optoelectronic Devices and Properties, InTech, pp. 323-348.

Cech, V. and J. Jevicky. (2013) Effectiveness of Passive Optoelectronic and Laser Rangefinders, in Proceedings of the 27th International Symposium on Ballistics. Editors: Wickert, M. - Salk, M., Freiburg, BRD, p. 251 - 262.

Cech, V. (2018) Multiple Stations Passive Optoelectronic Range-finding System, in Proceedings of the $1^{\text {st }}$ International Conference on Defence Technology. Editors: Feng, Ch. - Woodley, C. - Li, B. Beijing, China, p. $815-818$

Kraus, K. (2000) Photogrammetry. Volume 1 - Fundamentals and Standard Processes. Dümmler, Köln, 397 p.

Roggeman, M. C., Welsh, B. (1996) Imaging Through Turbulence. CRC Press. 